\title{
Análisis
}

\section{Una profesión en redefinición: visión desde el Col·legi Oficial de Bibliotecaris-Documentalistes de Catalunya}

\author{
Por Eulàlia Espinàs
}

\begin{abstract}
Resumen: Se presentan algunos datos acerca del perfil y las demandas de los profesionales de la información y la documentación recogidos a partir de un estudio realizado en el año 2007 y de las opiniones expresadas por profesionales en activo en los espacios de intercambio generados por el Col-legi Oficial de Bibliotecaris-Documentalistes de Catalunya. De ellos ha surgido una serie de reflexiones que han motivado el plan de actuaciones para los años 2007-2009. Asimismo se exponen los retos más relevantes que deben plantearse las asociaciones según la percepción del Col-legi, para poder tener un papel activo y de referencia en el entorno económico y social actual.

Palabras clave: Perfil profesional, Profesionales de la información y la documentación, Asociaciones profesionales, Col-legi Oficial de Bibliotecaris-Documentalistes de Catalunya, Estudios de profesionales.

Title: Redefining a profession: a view from the Col.legi Oficial de Bibliotecaris-Documentalistes de Catalunya

Abstract: A 2007 survey and the views expressed by professionals active in the discussions generated by the Col-legi Oficial de Bibliotecaris-Documentalistes de Catalunya provide relevant data on the current profile and the demands of information and documentation professionals. A number of thoughts have emerged that led to the plan of action for the years 2007-2009. The most significant challenges that, according to the perception of the Col-legi, associations should consider in order to take an active role and reference in the current economic and social environment are also presented.
\end{abstract}

Keywords: Professional profile, Information and documentation professionals, Professional associations, Col-legi Oficial de Bibliotecaris-Documentalistes de Catalunya, Professional studies.

Espinàs, Eulàlia. "Una profesión en redefinición: visión desde el Col·legi Oficial de Bibliotecaris-Documentalistes de Catalunya". En: El profesional de la información, 2008, mayojunio, v. 17, n. 3, pp. 311-316.

DOI: 10.3145/epi.2008.may.07

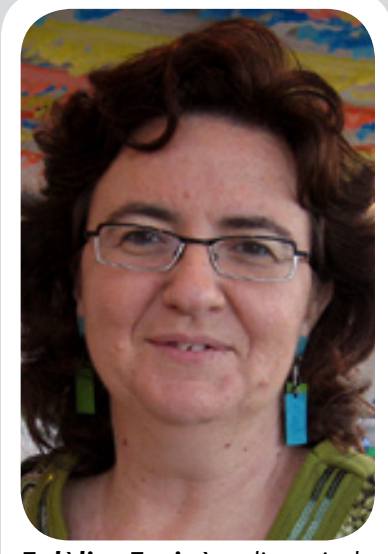

Eulàlia Espinàs, licenciada en biblioteconomía y documentación por la Universitat de Barcelona, ha trabajado en bibliotecas y centros de documentación en el proceso de su creación y puesta en funcionamiento. Es responsable de promoción cultural y evaluación de públicos del área de cooperación cultural de la Generalitat de Catalunya, profesora de políticas de información en la Universitat de Barcelona y Presidenta del Colllegi oficial de Bibliotecaris-Documentalistes de Catalunya.
MÁS ALLÁ DE LAS REFLEXIONES PERSONALES que cada uno de nosotros ha hecho a lo largo de su vida profesional, creemos que la experiencia de la actual junta directiva en el Col-legi Oficial de Bibliotecaris-Documentalistes de Catalunya (Cobdc), aunque breve, puede aportar algunos elementos de reflexión acerca de los cambios que se están viviendo entre los trabajadores de la información y de algunos de los retos que nos depara el presente y el futuro de la profesión.

\section{Perfil de los profesionales en Catalunya}

En la primavera de 2007 la empresa MetraSeis, especializada en estudios de opinión, realizó por encargo del Col-legi un estudio de valoración y expectativas de los profesionales del sector sobre la actividad y los servicios que éste ofrecía. La investigación, de carácter cuantitativo, se hizo a través de una entrevista telefónica a una muestra de 300 colegiados y 250 profesionales en activo no colegiados, además de 100 entrevistas personales a estudiantes de la universidad, lo que supone un total de 650 participantes.

Los resultados permiten extraer información relevante sobre algunos aspectos que caracterizan a bibliotecarios y documentalistas en la actualidad. En primer lugar hay que destacar que se mantiene una destacada presencia femenina: el $81,3 \%$ de colegiados son mujeres. Otro dato interesante son los años de experiencia en la profesión, con una media de 14,3 años los colegiados y de 10,8 los que no lo es- 
tán. En la distribución del lugar de trabajo destaca el vínculo entre los profesionales del sector público y la colegiación $(66,5 \%)$.

\section{"Se mantiene una destacada presencia femenina: el $81,3 \%$ de colegiados son mujeres"}

Se pueden hacer varias lecturas, pero destaca la confirmación que los profesionales del sector privado, coincidiendo con la concentración de los más jóvenes y de los ámbitos emergentes, presentan una baja presencia entre los colegiados (figura 1).

\section{Demandas formativas}

Una de las actividades más relevantes del Cobdc es su oferta formativa, que responde fundamentalmente a dos aspectos: la convicción de que es necesario un aprendizaje continuo a lo largo de la vida profesional si se desea dar respuesta a las nuevas necesidades que la sociedad nos plantea; y la demanda de los propios profesionales de orientar la formación hacia áreas no estrictamente académicas, pero muy necesarias para desenvolverse en el día a día laboral.

El estudio citado incluía un apartado de sugerencias o propuestas de mejora que el Cobdc debería ofrecer. Se han podido extraer las siguientes peticiones sobre la oferta formativa: que sea más amplia y diversificada de temas $(21,7 \%)$; descentralizada fuera de Barcelona ciudad (19\%); y lo más actualizada posible, incluyendo los temas más innovadores (18\%).

En los últimos cinco años (2002-2006) el Col-legi ha organizado 173 cursos en todo el territorio catalán, en los que han participado 3.662 personas y se ha hecho un esfuerzo por diversificar la oferta, con temáticas como:
"A lo largo de 2007 se ha implementado una plataforma virtual que permite ofrecer cursos en línea"

- análisis y representación de la información,

- colecciones y política documental,

- creación y difusión de contenidos,

- fuentes y recuperación de la información,

- gestión y organización de centros de información,

- interacción con los usuarios y clientes,

- marco jurídico y administrativo, etc.

Ante los datos que nos ha proporcionado el estudio, a partir del año 2007 se han empezado a intro-

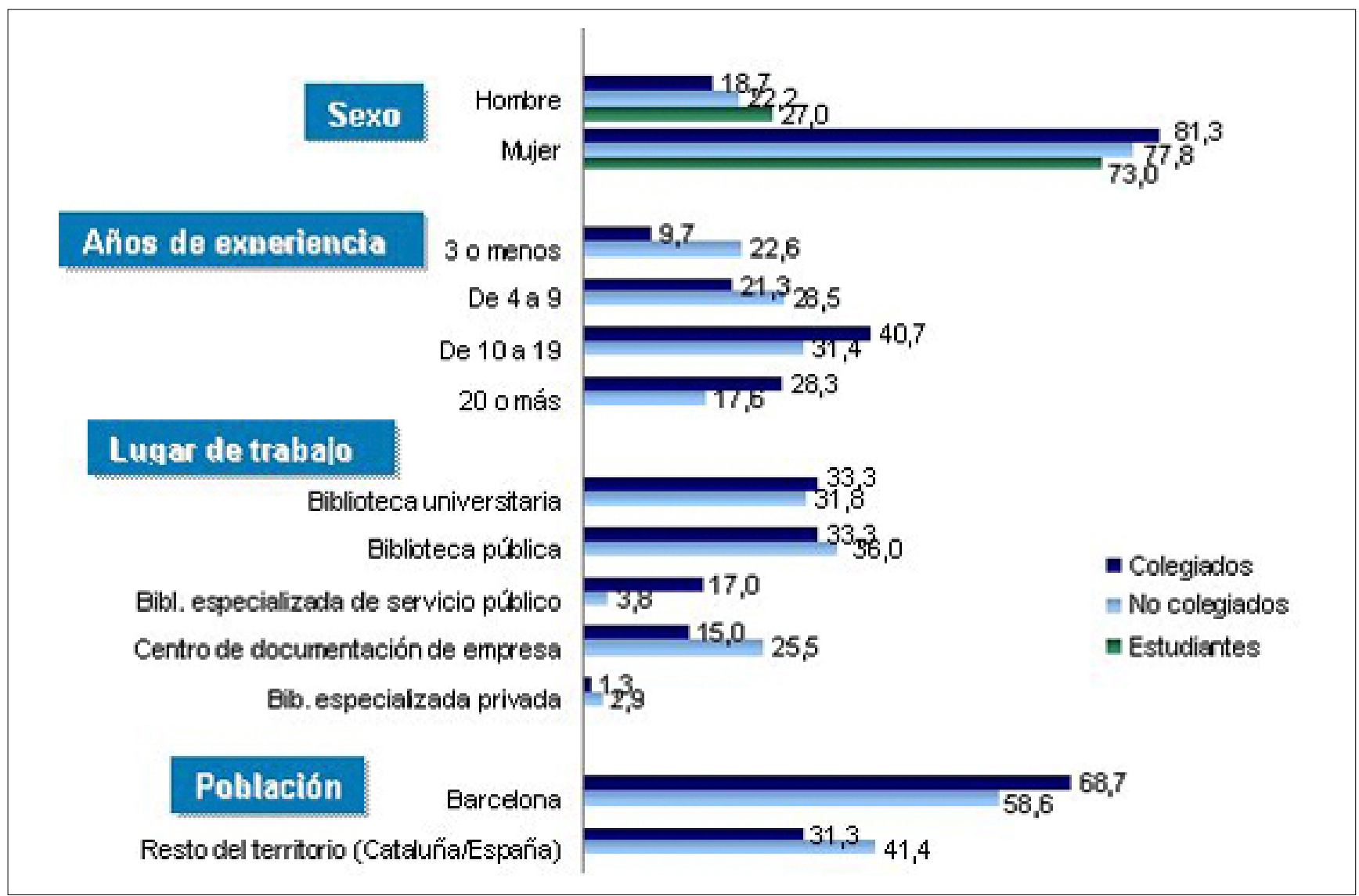

Figura 1 


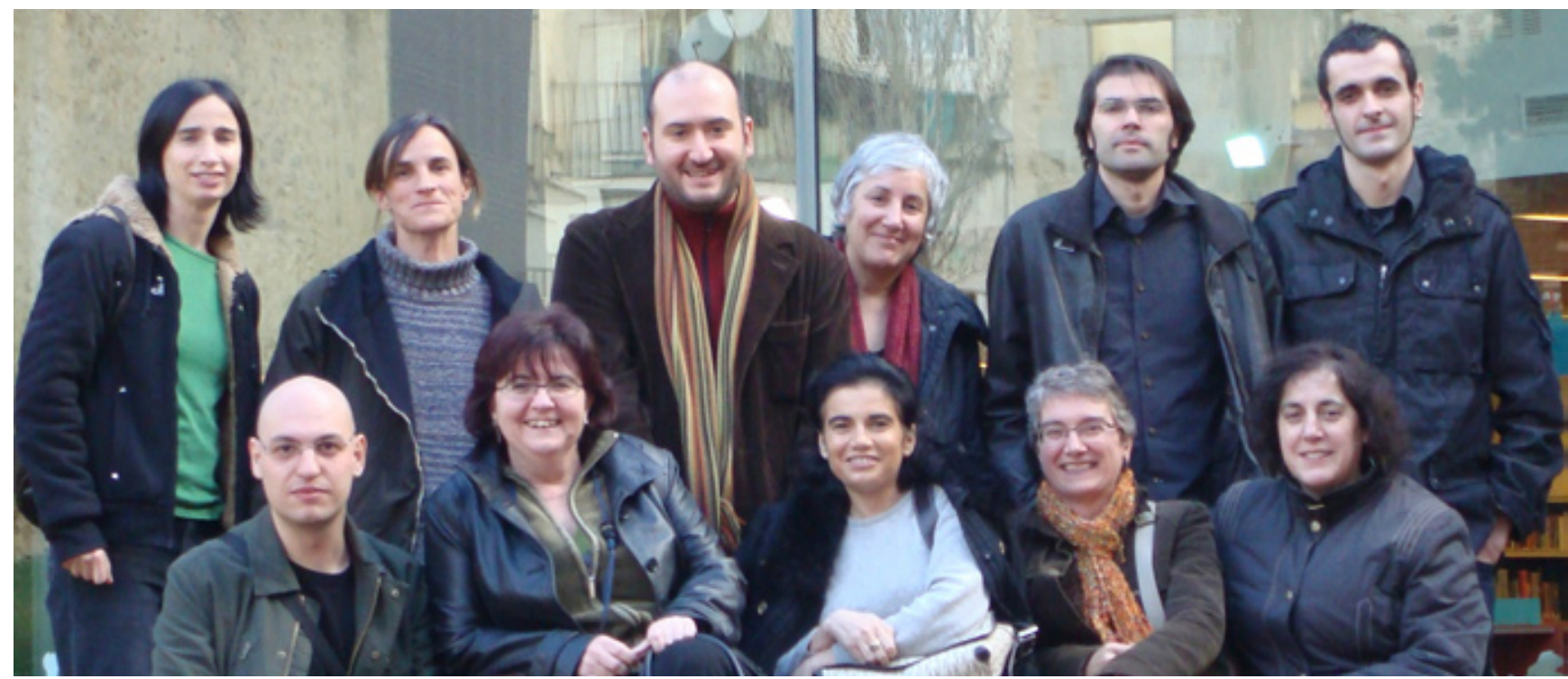

Los miembros de la Junta 2007-2009 del Col·legi son de diferentes especialidades, zonas geográficas y trayectorias. Nunca antes habían formado parte de una junta directiva y en ellos prima su profesionalidad y conocimiento: Carme Fenoll, Maite Comalat, Fidel Bellmunt, Núria Llebaria, Alfons Codina y Ferran Burguillos. Sentados: Javier Leiva, Eulàlia Espinàs, Laura Padró, Anna Rovira y Antònia Capdevila

\section{"Existe la necesidad de abrir la profesión a otros conocimientos a través de formación en temas colaterales a bibliotecas y documentación"}

ducir temas de otros entornos profesionales, formatos más variados (talleres, seminarios, etc.) y en horarios más diversificados, ofreciendo además respuesta a la demanda de múltiples instituciones que desean formación a medida.

Hemos apostado por la tecnología y a lo largo de 2007 se ha desarrollado una plataforma virtual que nos permite ofrecer cursos en línea, además de gestionar los cursos presenciales de una forma más ágil. A la espera de disponer del vaciado de encuestas de este último año podemos afirmar que se ha percibido una valoración muy positiva de esta nueva etapa.

\section{Aproximando el Col.legi a los profesionales}

La dispersión de bibliotecarios y documentalistas en el territorio hace que a menudo la relación directa no sea todo lo fluida que sería deseable. Aprovechando la ocasión de presentar una nueva actividad de participación, a lo largo del año 2007 se organizó un conjunto de encuentros que tomaron la forma de desayunos en cinco zonas del territorio catalán. Se convirtieron en un punto de encuentro muy vivo entre los profesionales y la junta directiva y de las aportaciones que se recogieron se pueden destacar las siguientes:

- Beneficiarse de los servicios del Col-legi sin tener que desplazarse a Barcelona; en este sentido se señalaron las posibilidades de los sistemas virtuales de comunicación y de trabajo.

- Abrir la profesión a otros conocimientos a través de formación en temas colaterales a las bibliotecas y a los servicios de información y documentación en general; organización de viajes profesionales; creación cooperativa de un banco de recursos y de buenas prácticas; elaboración de estudios comparativos sobre salarios, servicios, etc.

- Elaborar un estudio que defina las tareas y funciones de los profesionales del sector para poder afrontar la multiplicidad de tareas que se les exigen y el trabajo coordinado con otros profesionales.

Gracias a las opiniones recogidas en el estudio y a los encuentros desayuno, la junta estableció los objetivos y las actuaciones prioritarias a emprender a lo largo de su mandato, que deberán dar fruto a lo largo de 2008 y 2009.

\section{Participación en grupos}

Otro de los aspectos importantes a tener en cuenta en el análisis del sector es la capacidad de participación en grupos de trabajo, comisiones, consejos, etc. Aunque desde nuestra perspectiva no podemos ofrecer una visión general del tema, sí es posible aportar los datos de aquellos que el propio Col-legi impulsa.

Los grupos de trabajo del $\mathrm{Co}$ $b d c$, que nacen por iniciativa de los propios colegiados, tienen el objetivo de facilitar un espacio de intercambio entre profesionales con intereses comunes. Su diversidad se debe a múltiples aspectos, como

a) Especialización:

- gestión de la información en ciencias de la salud,

- bibliotecas de arte, 
- documentación musical,

- bibliotecas infantiles, etc.

b) Tipo de material o información:

- bibliotecas y cómics,

- reserva y libro antiguo,

- literatura fantástica y ciencia ficción,

- lectura fácil, o

- información y documentación gay, lésbica, bisexual y transgénero $(Q . d o c))$.

c) Ámbito territorial (Terres de l'Ebre), o

d) Procedimientos y programas

- gestión documental y de archivos,

- software libre para los profesionales de la información,

- usabilidad, etc.

Además de las reuniones periódicas que mantienen sus integrantes, así como los espacios virtuales de comunicación e intercambio que muchos de ellos han creado (wikis, blogs, etc.), las actividades que llevan a cabo contribuyen al dinamismo del Col.legi y facilitan puentes con otras profesiones. Algunos incluso organizan jornadas que se han consolidado más allá de los colegiados y de nuestro sector profesional. Este es el caso de las Jornadas de software libre y las de Usabilidad. Otros centran su actividad en la selección de materiales para bibliotecas, asesorando en temas especializados, en expurgos o en políticas de la colección de algunas instituciones, como es el caso del grupo Q.doc o el de Cómics.

El Col-legi ofrece soporte económico y logístico a esos grupos y favorece su participación en foros profesionales. Al mismo tiempo consideramos que deben cumplir con un mínimo de requisitos para que realmente su existencia tenga sentido en el seno del Cobdc por lo que actualmente se está revisando la normativa que rige su funcionamiento.

De todas formas, vista la variable actividad que presentan unos y otros, se observa que el apoyo que se les presta no es suficiente y se han iniciado acciones para difundir su existencia, fomentar la participación de un mayor número de miembros e impulsar un nivel más alto de actividad, facilitando herramientas virtuales para desarrollar su labor y garantizando su visibilidad en la web y en las publicaciones del Col.legi.

Por otro lado se impulsa la creación de comisiones y consejos formados por colegiados voluntarios con objeto de acompañar determinadas actuaciones de la junta directiva. El objetivo es ayudar a obtener una visión más global de determinados problemas o a trabajar más a fondo en temas concretos. En esta línea se pueden destacar el Consejo editorial de la revista Ítem, el Consejo asesor de la 11es Jornades catalanes d'informació i documentació, la Comisión deontológica o el equipo de revisión de la colección Directrices IFLA.

\section{Temas para el debate $y$ la reflexión}

En el Cobdc estamos inmersos en un proceso de reflexión sobre algunas cuestiones que afectan de lleno a los profesionales de la información. Consideramos que convendría debatir sobre los temas que exponemos a continuación para, si fuera necesario, organizarnos con el objetivo de no quedar relegados como profesión a un ámbito secundario ante los grandes retos de la sociedad de la información y del conocimiento.

\section{Diplomados/licenciados y nue- vas titulaciones}

Es conocida por todos la reforma que dentro de poco tiempo se introducirá en los estudios universitarios. Después del cambio que supuso la aprobación de la licenciatura en documentación en 1992 y las siguientes modificaciones en algunos de los planes de estudio de la diplomatura, este nuevo cambio proporciona una excelente oportunidad para reflexionar sobre la formación y las necesidades de los nuevos profesionales. Por otra parte pone en evidencia la creciente necesidad de ordenar el mercado laboral que no ha identificado como profesionales de pleno derecho a diplomados y licenciados como niveles diferenciados y con una cierta especialización.

\section{"Existe la creciente necesidad de ordenar el mercado laboral que no ha reconocido a diplomados y licenciados como niveles diferenciados y con una cierta especialización"}

Desde hace tiempo el Cobdc informa a empresas y administraciones sobre el hecho de disponer de titulaciones específicas aceptadas en dos niveles, que es necesario respetar en el momento de contratar y establecer la remuneración.

La entrada de las nuevas titulaciones en el marco del Espacio Europeo de Educación Superior que pretende armonizar los planes de formación europeos implica un único título de grado de 4 años y elimina la diferencia de los dos grados actuales como formación inicial. Las nuevas titulaciones se deberán aplicar en todas las universidades españolas y está previsto que entren en vigor en el curso 2009-2010, aunque algunas universidades ya han presentado propuestas para introducirlas en el período 2008-2009.

Diferentes colectivos profesionales participaron en la revisión de 
la propuesta que en su momento hicieron las universidades. Catalunya no ha sido una excepción y el Col-legi participa de forma activa y permanente en este proceso de colaboración y asesoramiento en la nueva propuesta, ya que consideramos que ésta es una muy buena iniciativa en la que todos debemos implicarnos para garantizar que la reflexión contemple todos los ámbitos profesionales del futuro y que el presente ya apunta.

Este cambio pondrá en evidencia la necesidad de establecer equivalencias entre titulaciones, especialmente importante en la función pública, y aquí deberemos estar atentos a la oportunidad para poder argumentar la necesidad de reconocer no sólo la titulación sino las competencias de cada grupo profesional.

\section{Reconocimiento, remuneracio- nes y transversalidad}

Los bibliotecarios y documentalistas vinculados al sector público continúan siendo una parte muy importante de nuestro sector. La gran sorpresa posiblemente sea la poca evolución que ha experimentado este sector en lo que se refiere a la distinción de los profesionales.

En Catalunya pocas administraciones públicas han admitido el nivel de licenciado a quienes disponen de la licenciatura, incluso en aquellos casos en los que éstos ocupan cargos de responsabilidad, ni tampoco en las convocatorias de nuevas plazas. En este sentido el Col-legi está trabajando desde hace tiempo para que las administraciones lo tengan en cuenta, pero siempre ha servido de excusa una circunstancia previa: en muchas de ellas ni tan sólo reconocen la especialización en biblioteconomía como requisito previo para ejercer. Aun así se continúa informando a todos aquellos que realizan convocatorias sin los requisitos mínimos indispensables y se inician procesos judiciales por parte del Col-legi siempre que la situación sea legalmente defendible.

\section{"El análisis de las ofertas laborales nos permite detectar una lenta transformación en el perfil y la valoración que se nos atribuye"}

El análisis de las ofertas laborales nos permite detectar una lenta transformación en el perfil y la valoración de los profesionales, visible a través de la demanda de múltiples empresas y de lugares de trabajo con perfiles más variados y poco clasificables. Llegar a todos es un reto no fácil de cumplir; por eso muchas veces el Cobdc ha optado por campañas indirectas, ya sean publicitarias, estando presentes en entornos no estrictamente bibliotecarios, participando en grupos de trabajo, comisiones o reuniones externas a la propia profesión. Somos conscientes que éste es uno de los aspectos en los que haría falta una mayor actividad y presencia si realmente deseamos incidir y ser considerados en el mercado laboral como profesionales expertos más allá de los servicios estrictamente bibliotecarios.

Las nuevas profesiones que está haciendo emerger la sociedad de la información están estrechamente vinculadas a los profesionales de la información y se les está ofreciendo nuevas posibilidades, tanto en empresas de servicios y de gestión de contenidos como simplemente abriendo oportunidades a emprendedores para la creación de su propia empresa. De todas formas, no es una situación reconocida abiertamente y esto provoca que a veces se deba entrar en competencia con otros sectores y tener que explicitar lo que para nosotros es obvio.
Encontramos cada vez más diplomados desarrollando sitios web institucionales, organizando actividades culturales o colaborando en proyectos de investigación científica. De la misma forma descubrimos historiadores, periodistas, informáticos, etc., realizando tareas propias de un documentalista. Todos ellos opinan que no son intrusos en otros sectores y que posiblemente el atractivo del lugar de trabajo se encuentra precisamente en el hecho de estar en la frontera y en la interrelación con otras profesiones. Entendemos que ello es una oportunidad más que una amenaza, si bien no todos los profesionales lo perciben así, por lo que observamos que éste es otro de los largos caminos que debemos recorrer con cierta celeridad.

\section{Colegios y asociaciones ¿para qué?}

Es necesario que seamos reconocidos con unos mínimos indispensables de titulación y remuneración económica. Y muy posiblemente habría situaciones negativas que se evitarían si la colegiación fuera considerada como un requisito indispensable para el ejercicio de la profesión. Sin esto, la ley del libre mercado y la libre competencia hace que los mínimos indispensables sean difíciles de hacer cumplir y que muchos bibliotecarios y documentalistas tengan complicado hacer valer el nivel de sus conocimientos y sus capacidades. Pero éste es un requisito que queda muy lejos de las posibilidades inmediatas, que no garantiza de forma automática una mejor valoración o reconocimiento, ni tampoco una mayor capacidad por parte de los titulados del sector para desarrollar las tareas y funciones que actualmente se les requieren.

Para las asociaciones el reto es hacer visible este apreciación ante las instituciones, ante las empresas y ante la propia administración. Las asociaciones más que nunca debemos: 
